\title{
Dietary Modification Trial in Community-Dwelling Japanese Elderly: A Pilot Study
}

\author{
Chika Momoki $^{\mathrm{a},}$,, Taeko Tsuji ${ }^{\mathrm{b}}$, Yukina Shikata ${ }^{\mathrm{b}}$, Hana Urade $^{\mathrm{b}}$, Hideki Morimoto ${ }^{\mathrm{c}}$, \\ Shinya Nakajimad ${ }^{\text {, Daiki Habu }}{ }^{\mathrm{b}}$
}

\begin{abstract}
Background: This study examined the effects of 6-month nutrition education programs for community-dwelling elderly.

Methods: This study enrolled 50 community-dwelling elderly who regularly visit outpatient clinics. The programs had three goals: salt reduction, increase in dietary fiber, and adequate protein intake. Since it would be difficult for elderly to achieve all goals concurrently, a single goal was chosen by participants themselves. Anthropometric measurements, blood sampling, and assessment of dietary intake were performed at baseline, 3 months, and 6 months.
\end{abstract}

Results: The nutrition education program for salt reduction was well accepted by the participants and the amount of daily salt intake showed median value of $9.6 \mathrm{~g}$ at baseline, $8.0 \mathrm{~g}$ at 3 months and 8.1 $\mathrm{g}$ at 6 months $(\mathrm{P}=0.005)$. The amount of dietary fiber intake only slightly increased after taking the nutrition program (median value of $13.4 \mathrm{~g}$ at baseline, $15.3 \mathrm{~g}$ at 3 months and $15.5 \mathrm{~g}$ at 6 months; $\mathrm{P}=$ 0.695), because of difficulties in introducing new food options to the diet. After taking the adequate protein intake program, participants showed small decreases in protein (a modification from $1.24 \mathrm{~g} / \mathrm{kg}$ IBW to $1.20 \mathrm{~g} / \mathrm{kg} \mathrm{IBW}$ ) and salt intake ( 8.2 to $7.3 \mathrm{~g}$ ) at 3 months, but the effects were not sustained at 6 months.

Conclusion: This nutrition education program focusing on a single nutrient may serve as a strategy to successfully reduce salt intake and

Manuscript accepted for publication May 02, 2017

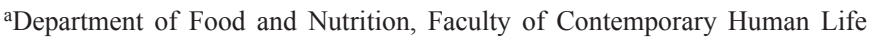
Science, Tezukayama University, 3-1-3 Gakuenminami, Nara-shi, Nara 6318585, Japan

bDepartment of Medical Nutrition, Graduate School of Life Science, Osaka City University, 3-3-138 Sugimoto, Sumiyoshi-ku Osaka-shi, Osaka 5588585, Japan

${ }^{\mathrm{c}}$ Morimoto Naika Clinic, 1-19-20 Yata, Higashisumiyoshi-ku Osaka-shi, Osaka 546-0023, Japan

dNakajima Clinic, 2-1-1-133 Asahimachi, Abeno-ku Osaka-shi, Osaka 5450051, Japan

${ }^{\mathrm{e} C}$ Corresponding Author: Chika Momoki, Department of Food and Nutrition, Faculty of Contemporary Human Life Science, Tezukayama University, 3-1-3 Gakuenminami, Nara-shi, Nara 631-8585, Japan.

Email:momoki@tezukayama-u.ac.jp

doi: https://doi.org/10.14740/jocmr3052w improve systolic blood pressure control in community-dwelling elderly individuals who regularly visit outpatient clinics. In our view, dietary and lifestyle habits should be taken into account as much as possible in nutrition education for elderly individuals.

Keywords: Dietary counseling; Nutrition education; Nutritional status; Elderly; Outpatient clinic

\section{Introduction}

The extension of healthy life expectancy is a challenging issue in super-aged societies. Many developed countries facing aging populations are attempting to prolong the independence of the elderly at home [1]. There is a growing understanding that maintenance of a healthy lifestyle is important to increase healthy life expectancy. In particular, maintaining adequate dietary intake is essential for preserving health [2-4].

However, lifestyle modification, especially dietary modification, is often difficult for elderly individuals [5]. This is largely because dietary habits are deeply rooted in the lives and culture of elderly individuals. Additionally, there is a tendency to consume decreased amounts and variety of foods with age [6].

We developed nutrition education programs to achieve a healthy lifestyle with minimal dietary modification and tested their efficacy in a group of community-dwelling elderly individuals, using several new strategies. First, we conducted the current study in two outpatient local clinics visited by many elderly clients on a regular basis. We hypothesized that effective intervention could be performed in these outpatient clinics as most clients live within walking distance of the clinic, have good relationships with the physicians, and are prepared to accept advice regarding diet. We also anticipated that detailed and frequent advice would be available in such a setting.

Second, we defined three nutritional goals: 1) salt reduction, 2) increase in dietary fiber, and 3 ) adequate protein intake. Study participants were independent community-dwelling elderly individuals without serious diseases. Most participants had a mild lifestyle-related disease for which medications were prescribed. With the exception of some patients requiring specific nutrition management, such as those with type 2 diabetes mellitus and chronic kidney disease, the participants 
had dietary problems commonly observed among the Japanese elderly population (reported in the National Health and Nutrition Survey): 1) excessive salt intake, 2) insufficient dietary fiber intake, and 3) excessive protein intake (for those with diminished renal function). Since it would be difficult for elderly people to achieve all three nutritional study goals at the same time, one goal was chosen for the 6-month nutrition education programs in this study. The goal was chosen by participants after discussions with healthcare professionals. We postulated that choosing their own goal would motivate the participants and result in a higher success rate.

Dietary modification is often difficult for communitydwelling elderly individuals. This study examined the effects of our relatively intense nutrition education programs (conducted by physicians and registered dieticians, with a nutritional goal chosen by participants) on dietary intake in community-dwelling elderly individuals.

\section{Materials and Methods}

\section{Study design and subjects}

This was an open cohort study which examined the effects of 6-month nutrition education programs for 50 communitydwelling elderly individuals. Participants were selected from among 60 individuals aged 65 years or above who regularly visited one of the two clinics between July 2011 and May 2014. The following patients were excluded from the study: one patient with mild cognitive impairment and a communication disorder and nine patients who did not visit the clinic after the first session. A total of 50 participants (15 men and 35 women with a mean age of $74.0 \pm 5.5$ years) were included in the study after informed consent was obtained from the participants and attending physicians. The incidence of primary diseases in this cohort was as follows: type 2 diabetes mellitus, 12 patients $(24 \%)$; dyslipidemia, 11 patients $(22 \%)$; renal dysfunction, 11 patients $(22 \%)$; liver dysfunction, seven patients (14\%); hypertension, seven patients (14\%); and other diseases, two patients $(4 \%)$. This study complied with the principles of the Declaration of Helsinki, and written informed consent was obtained from all participants.

\section{Dietary counseling}

The primary goal of dietary counseling was determined by the participants in collaboration with the physicians and registered dieticians, after considering the disease, nutritional status, and dietary intake of the participants. Participants were asked to choose one of the three goals (salt reduction, increase in dietary fiber, or adequate intake of protein (only for those with diminished renal function)) based on their needs and the feasibility of the goals. Accordingly, participants were classified into the salt reduction group $(n=18)$, the increase in dietary fiber group (dietary fiber group) $(n=21)$, or the adequate intake of protein group (protein reduction group) $(n=11)$. The first session took place on the day of enrolment, with participants providing informed consent after explanation of the protocol by the physician. A dietary intake assessment of participants was performed, and dietary counselling was provided by the dieticians. Follow-up assessments were conducted at approximately 1 (initiation of dietary counselling), 3, and 6 months (Fig. 1).

The instructions for each program were described as follows. Participants in the salt reduction group were advised to gradually reduce the frequency of intake of foods that may contribute to an increase in their salt intake. Participants in the dietary fiber group were encouraged to increase their intake of whole grains (as their staple food), vegetables, seaweed, and mushrooms. Participants in the protein reduction group received nutrition counseling according to the Dietary Recommendations for Chronic Kidney Disease [7].

\section{Measurements}

All participants received four or more assessment or counseling sessions: session one (baseline), session two (initiation of dietary counseling), session three at 3 months, and session four at 6 months. Additional sessions were performed as needed. During the first session, a nutritional assessment, anthropometric measurements, and a daily activity assessment were performed, and a questionnaire regarding daily life was administered. A history of past and present illness, information regarding medication, and blood biochemistry test results were obtained from the clinics. For each dietary counseling session, participants were instructed to perform a dietary record for 2 days (nonconsecutive) using a photo method, food recording method, and a physical activity record over the following week using the Lifecorder Gs (Suzuken Co., Ltd, Nagoya, Japan). At each session, participants also underwent anthropometric measurements. The counseling content was based on the evaluation of food intake and physical activity, calculated using a nutrition dietary assessment software (Nutrition Meister Ver. 2.0 and Ver. 3.0, Access Intelligence LLC, Rockville, MD, USA).

\section{Questionnaire}

Nutritional status on session one (baseline) was assessed using the $\mathrm{MNA}^{\circledR}$-SF (mini-nutritional assessment short form) (Nestle Japan Co., Ltd, Tokyo, Japan). Daily activity was assessed using the Tokyo Metropolitan Institute of Gerontology (TMIG) Index of Competence [8]. The TMIG Index of Competence is composed of 13 questions with three subscales: instrumental self-maintenance, intellectual activity, and social role subscales. Each question is worth 1 point, for a total of 13 points, and a higher score indicates a higher level of activity in daily life. The TMIG Index of Competence was self-administered by the participants.

\section{Anthropometric measurements}

Body height and body weight were measured by the examiner at the clinic. Body fat percentage was measured using a body 


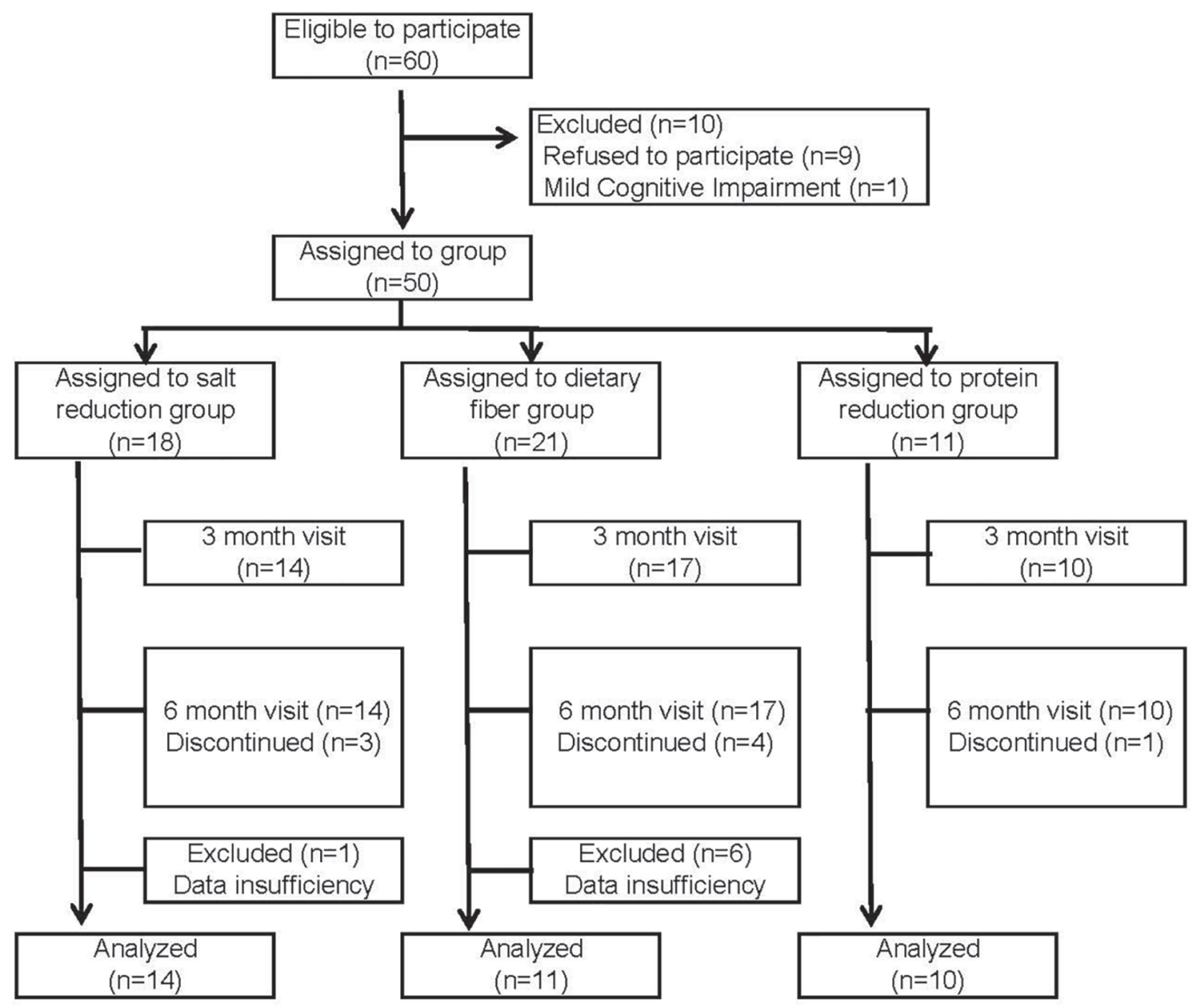

Figure 1. Flow diagram according to selection and non-continuation.

composition balance meter (EW-FA70P, Panasonic Co., Ltd, Osaka, Japan).

\section{Statistical analysis}

Statistical analysis was performed using SPSS ver. 22.0 (SPSS Inc., IBM Corp., Armonk, NY, USA). Comparison of continuous variables among the three groups was performed using the Kruskal-Wallis test. Comparison of nominal variables was performed using the Chi-square test. Comparison of data over time was performed using the Friedman test. The Bonferroni correction was applied when statistical significance was observed among the three groups. In principle, the data were shown as medians, 25th, and 75th percentiles, while some were shown as medians and minimum and maximum values due to the small sample size.

\section{Results}

\section{Characteristics}

Table 1 shows the characteristics and baseline parameters of all groups. The median body mass index (BMI) of each group was
$24 \mathrm{~kg} / \mathrm{m}^{2}$, with severe malnutrition not observed in any participants. Individuals in the protein reduction group were slightly older compared with those in the other two groups. Members of this group also had higher body fat percentage and lower total energy expenditure and number of steps compared with the other two groups. There were no significant differences in other anthropometric measures among the three groups. Of the 50 participants, the numbers completing 6 months of followup were 14 participants in the salt reduction group, 11 in the dietary fiber group, and 10 in the protein reduction group.

\section{Comparison over time in the salt reduction group}

Table 2 shows the changes at 3 and 6 months in the salt reduction group. BMI showed no changes over the 6-month follow-up period, while the body fat percentage significantly decreased. Biochemistry tests showed an improvement in neutral fat. Evaluation of nutritional intake showed that the ratio of protein intake to total energy intake slightly decreased, while that of carbohydrate intake to total energy intake significantly increased. Salt intake significantly decreased from baseline to 6 months: median value of $9.6 \mathrm{~g}$ at baseline, $8.0 \mathrm{~g}$ at 3 months and $8.1 \mathrm{~g}$ at 6 months $(\mathrm{P}=0.005)$. Systolic blood pressure showed a tendency to decrease over time: median value of $135 \mathrm{~mm} \mathrm{Hg}$ at baseline, $122 \mathrm{~mm} \mathrm{Hg}$ at 3 months and 
Table 1. Characteristics and Baseline Parameters of All Groups

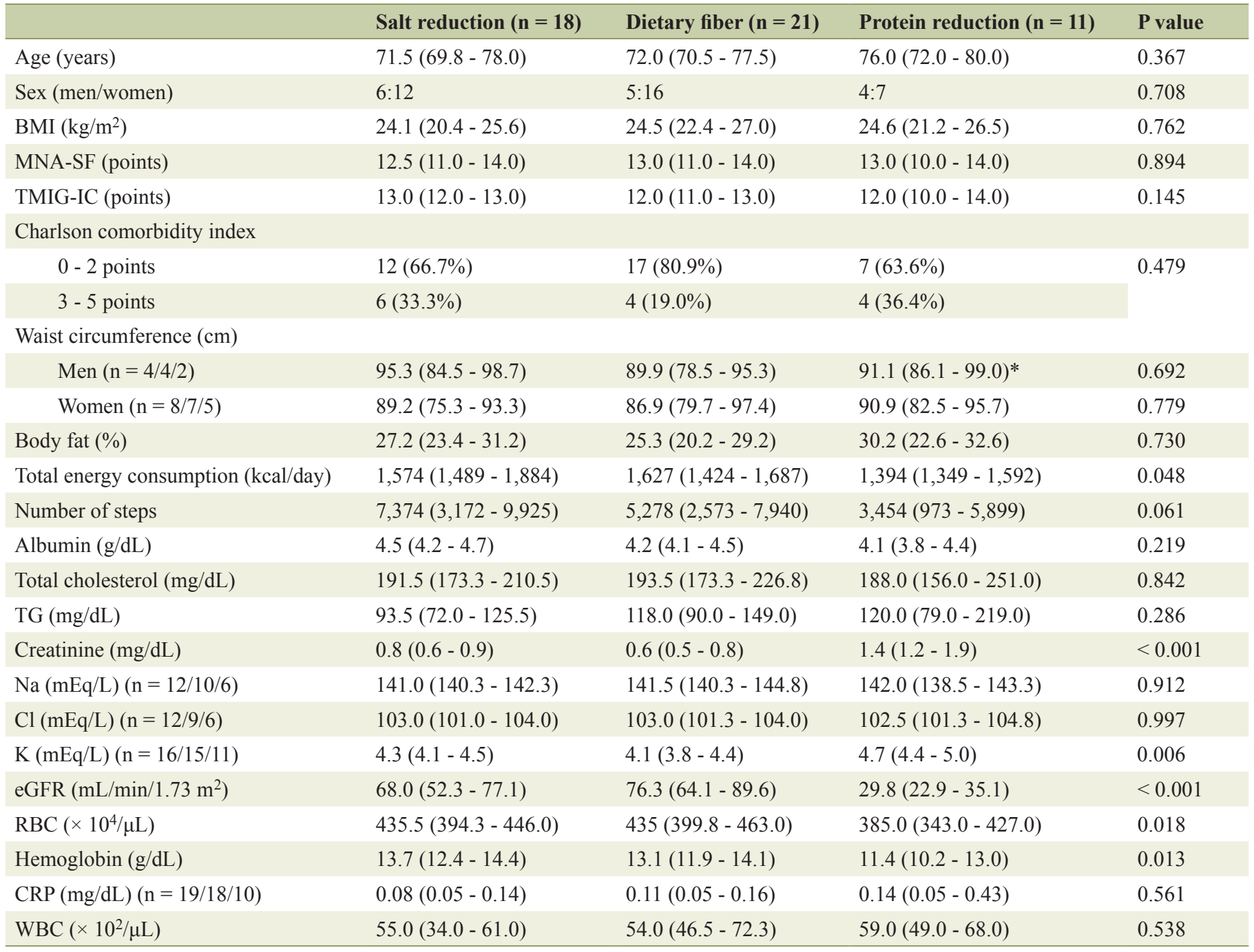

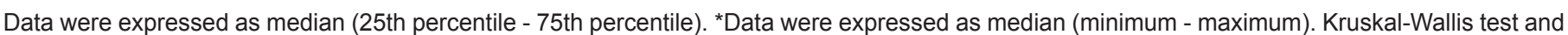
Chi-square test for categorical variables were performed for comparison of three groups. MNA-SF: mini-nutritional assessment short form; TMIG-IC: Tokyo Metropolitan Institute of Gerontology Index of Competence; TG: triglyceride; eGFR: estimated glomerular filtration rate; RBC: red blood cell count; WBC: white blood cell count.

$125 \mathrm{~mm} \mathrm{Hg}$ at 6 months $(\mathrm{P}=0.085)$. Moreover, blood pressure control improved, although the change was not statistically significant.

\section{Comparison over time in the dietary fiber group}

Table 3 displays the changes at 3 and 6 months in the dietary fiber group. Anthropometric measurements did not demonstrate significant changes from baseline to 6 months. Evaluation of nutritional intake showed that energy intake per $\mathrm{kg}$ of ideal body weight decreased from 32.2 to $28.8 \mathrm{kcal} / \mathrm{kg} / \mathrm{day}$, and protein intake per $\mathrm{kg}$ of ideal body weight decreased from 1.30 to $1.19 \mathrm{~g} / \mathrm{kg} /$ day. Salt intake also decreased from median value of $9.4 \mathrm{~g}$ at baseline to $7.3 \mathrm{~g}$ at 6 months $(\mathrm{P}=0.078)$. Dietary fiber intake slightly increased from median value of 13.4 $\mathrm{g}$ at baseline to $15.5 \mathrm{~g}$ at 6 months, although the change was not statistically significant $(\mathrm{P}=0.695)$.

\section{Comparison over time in the protein reduction group}

Table 4 displays the changes in the protein reduction group at 3 and 6 months. Serum potassium slightly decreased from baseline to 6 months. There were no notable changes in other biochemical parameters. Evaluation of nutritional intake showed that energy intake per $\mathrm{kg}$ of ideal body weight significantly decreased from baseline to 6 months. Protein intake per kg of ideal body weight and salt intake displayed slight improvements from baseline to 3 months, while intake slightly increased from 3 to 6 months. Moreover, after taking the adequate protein intake program, participants showed no decrease 
Table 2. Change in Variables at 3 and 6 Months in the Salt Reduction Group $(n=14)$

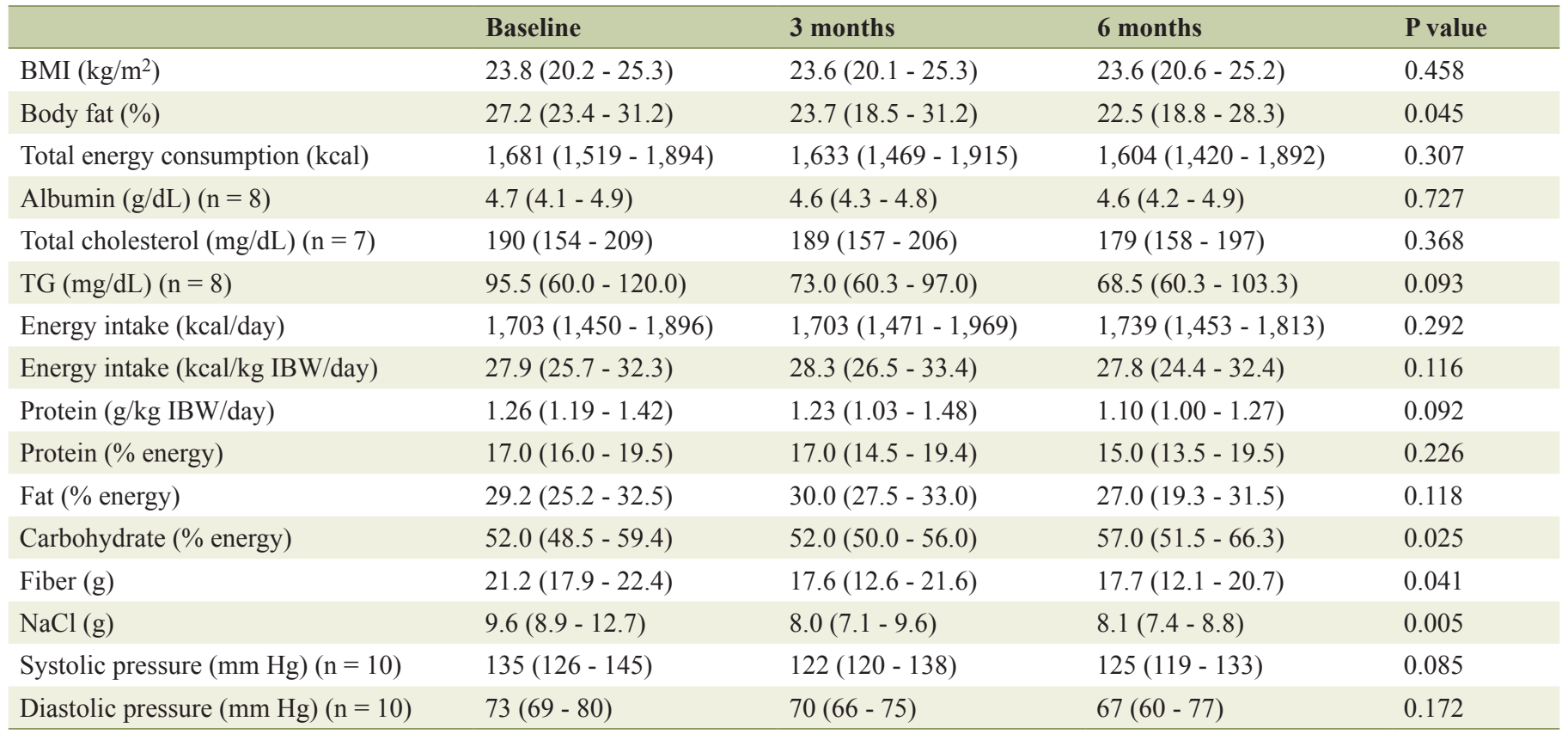

Data were expressed as median (25th percentile - 75th percentile). Friedman tests were performed for comparison of 3 time point. TG: triglyceride.

in estimated glomerular filtration rate (eGFR).

\section{Discussion}

This study examined the effects of 6-month nutrition education programs targeting the intake of a single nutrient in com- munity-dwelling elderly individuals. Among the three groups (dietary fiber increase, salt reduction, and protein reduction groups), participants in the salt reduction group showed significant improvement in the study targets following the program. However, participants in the dietary fiber group and the protein reduction group did not show notable changes because these programs required major changes in dietary patterns. In ap-

Table 3. Change in Variables at 3 and 6 Months in the Dietary Fiber Group $(n=11)$

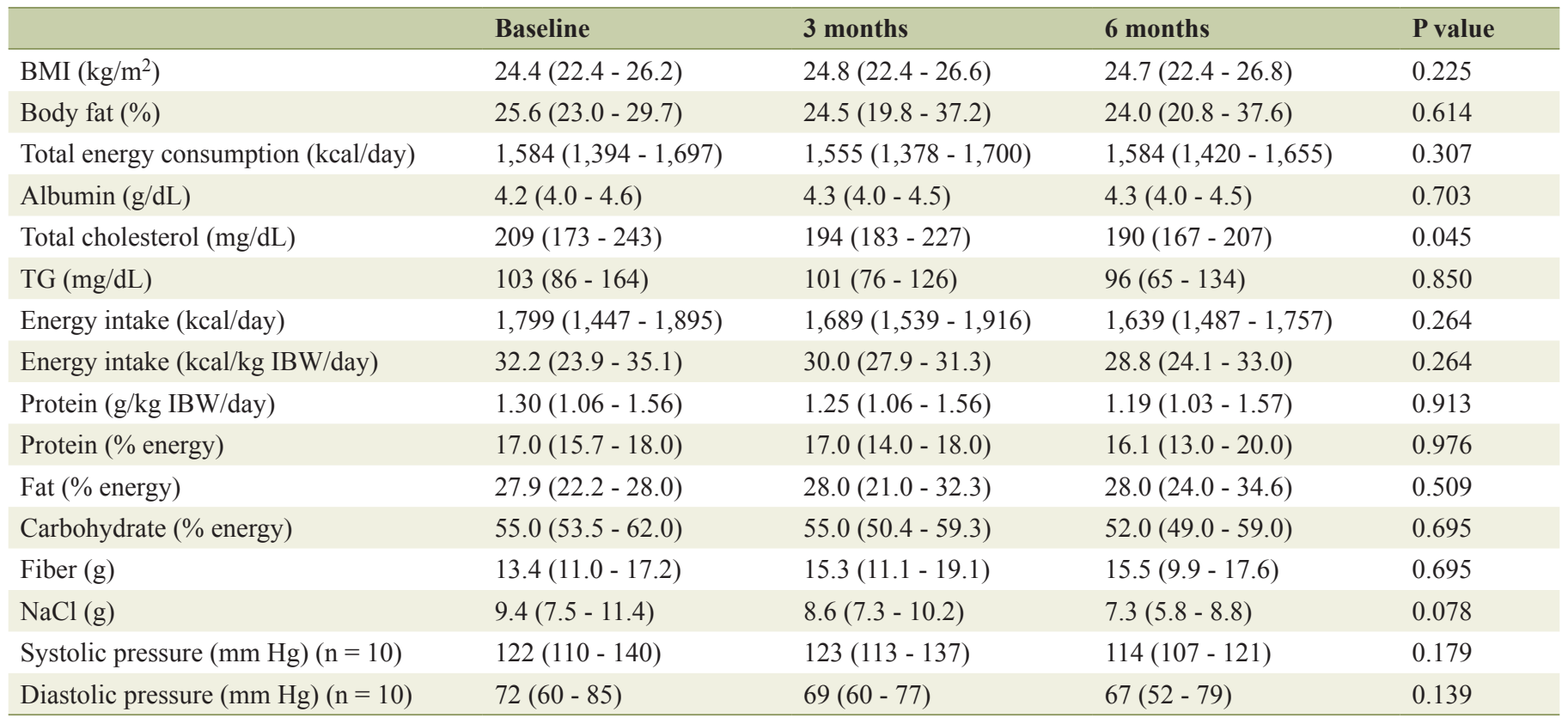

Data were expressed as median (25th percentile - 75th percentile). Friedman tests were performed for comparison of 3 time point. TG: triglyceride. 
Table 4. Change in Variables at 3 and 6 Months in the Protein Reduction Group $(n=10)$

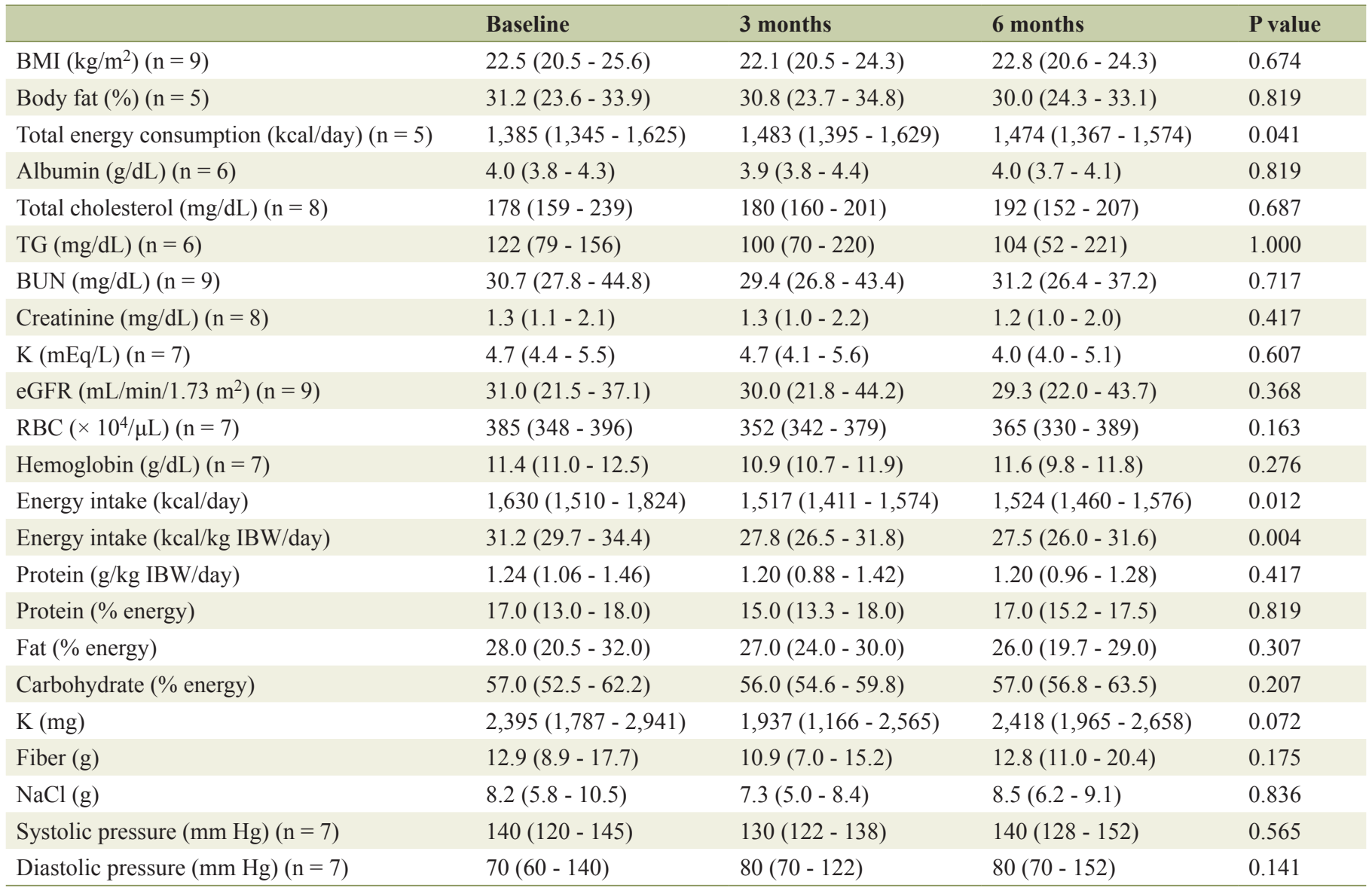

Data were expressed as median (25th percentile - 75th percentile). Friedman tests were performed for comparison of 3 time point. TG: triglyceride; BUN: blood urea nitrogen; eGFR: estimated glomerular filtration rate.

proximately $10 \%$ of participants, intake of energy and protein at baseline were slightly in excess although these improved to almost normal levels in 6 months. The results of the current study showed that our participant-oriented nutrition education program was partially effective.

Salt reduction was successfully achieved by participants in this nutrition education program. We believe that the salt reduction strategies, such as altering the seasoning of food and reducing the amount of soup consumed, were relatively easy to implement and well accepted by elderly individuals. Furthermore, participants who were able to appreciate the effect of salt reduction on lowering systolic blood pressure may have been more motivated during the program. A reduction of salt intake was also observed in the dietary fiber group. This finding may be because the education program was not strictly restricted to the nutrient chosen, and participants also received general dietary advice.

In the dietary fiber group, the intake of dietary fiber slightly increased, but did not reach the target values (19 $\mathrm{g}$ for men and $17 \mathrm{~g}$ for women) recommended by the Dietary Reference Intakes for Japanese (2015 edition) [9]. An epidemiological study reported that mean dietary fiber intake from fruits and vegetables in Caucasian women increased with age, from 12 $\mathrm{g}$ in women in their $20 \mathrm{~s}$ to $15 \mathrm{~g}$ in women in their $60 \mathrm{~s}$ and $70 \mathrm{~s}$ [10]. These findings were comparable to those of the present study. We advised study participants to consume low glycemic index staple foods and increase their intake of tubers and roots, seaweed, and various mushrooms to increase dietary fiber intake. However, participants or their families were reluctant to introduce new foodstuffs to their diet. In fact, many participants were not eating vegetables. In some cases, oral or dental problems may have influenced their dietary behavior. Furthermore, a considerable number of participants reported that they did not know how to cook seaweed or mushrooms. In our view, taking supplements may be beneficial to those who are unable to achieve dietary fibre goals through diet alone.

For the protein reduction group, energy and protein requirements were estimated on an individual basis according to the Dietary Recommendations for Chronic Kidney Disease [7], except in the case of a few participants. Based on these estimates, the recommended daily intake of each foodstuff was calculated, and a list was provided in written form to each participant and used in the dietary counseling sessions. The intake of protein and salt was found to have slightly improved at 3 months, but returned to baseline levels at 6 months. There was no significant decrease in the eGFR after 6 months. Moreo- 
ver, there was considerable effect in the maintenance of renal function. We believe that compliance with the dietary therapy based on the Dietary Recommendations for Chronic Kidney Disease may have been difficult for elderly people who preferred Japanese foods and fish. Dietary counseling was provided more frequently (at an interval of 1 or 2 months) for the majority of participants in this group. Following the program, many participants reduced both their protein intake and energy intake. We recommended the consumption of some good-tasting therapeutic foods (for snacking between meals) or therapeutic sweeteners for participants who had difficulty in changing their dietary patterns. However, these individuals did not achieve sufficient energy intake, most likely as a result of taste preferences or economic factors. A previous study reported that a nutrition education program led to increased intake of vegetables in diabetic patients at 3 months, but the effect was not maintained at 12 months [11], suggesting that maintaining the effect is much more difficult than simply achieving it. Conversely, another study reported that high-frequency telephone counseling was as effective as face-to-face counseling for lifestyle modification [12]. There is also a study that reported that an intense nutrition education program (including four different actions) contributed to reducing protein intake [13]. These reports suggest that more frequent follow-up might be needed for our programs. We also considered that the lack of significant effects in our study participants might be partly attributable to their insufficient understanding of the need to reduce protein intake, as participants did not have subjective symptoms of renal dysfunction. This rationale is supported by evidence that people requiring dietary modification for medical reasons tend to have nutritional knowledge and that elderly individuals are likely to accept nutrition education programs that are directly related to their health [14].

Nutrition education providers targeting elderly individuals should be aware that these individuals may have insufficient understanding of nutrition and that they are often reluctant to change their lifestyle habits. We believe that the dietary and lifestyle habits of the elderly should be respected as much as possible, except in cases of patients requiring strict dietary control of diseases. In principle, nutrition education for elderly individuals should be simple and easy [5].

The current study has several limitations. First, the sample size was small and the study area was limited, making the results of this study difficult to generalize to wider elderly. Second, there may have been differences in the levels of expertise among the dietitians who provided dietary counseling. Third, the influence of medications was not considered in this study.

\section{Conclusions}

This nutrition education program focusing on a single nutrient may serve as a strategy to successfully reduce the intake of salt and improve systolic blood pressure control for communitydwelling elderly individuals who regularly visited outpatient clinics. In addition, there was no significant decrease in the eGFR after 6 months; moreover, there was considerable effect in the maintenance of renal function, implying that the nutri- tion education program was effective. We believe that dietary and lifestyle habits should be taken into account as much as possible in nutrition education for elderly individuals.

\section{Acknowledgments}

We are grateful to the individuals and the clinic staff who participated in this study.

\section{Conflicts of Interest}

The authors declare that there are no conflicts of interest regarding the publication of this paper.

\section{Author Contributions}

Study conception and design: Chika Momoki and Daiki Habu. Acquisition: Hideki Morimoto and Shinya Nakajima. Acquisition, analysis and interpretation of data: Chika Momoki, Taeko Tsuji, Yukina Shikata, and Hana Urade. Critical revision: Daiki Habu.

\section{References}

1. Mathers CD, Sadana R, Salomon JA, Murray CJ, Lopez AD. Healthy life expectancy in 191 countries, 1999. Lancet. 2001;357(9269):1685-1691.

2. Fishman P. Healthy People 2000: what progress toward better nutrition? Geriatrics. 1996;51(4):38-42.

3. Drewnowski A, Evans WJ. Nutrition, physical activity, and quality of life in older adults: summary. J Gerontol A Biol Sci Med Sci. 2001;56(Spec No 2):89-94.

4. Haveman-Nies A, de Groot LC, van Staveren WA. Dietary quality, lifestyle factors and healthy ageing in Europe: the SENECA study. Age Ageing. 2003;32(4):427434.

5. Chernoff R. Nutrition and health promotion in older adults. J Gerontol A Biol Sci Med Sci. 2001;56(Spec No 2):47-53.

6. Morley JE. Decreased food intake with aging. J Gerontol A Biol Sci Med Sci. 2001;56(Spec No 2):81-88.

7. Japanese Society of Nephrology: Dietary recommendation of chronic kidney disease 2007. Nihon jinzo gakkai shi. 2007;49(8):871-878.

8. Koyano W, Hashimoto M, Fukawa T, Shibata H, Gunji A. [Functional capacity of the elderly: measurement by the TMIG Index of Competence]. Nihon Koshu Eisei Zasshi. 1993;40(6):468-474.

9. Ministry of Health, Labour and Welfare, Japan. Dietary Reference Intakes for Japanese, 2015. Tokyo: DaiichiShuppan Co, 2014 (in Japanese).

10. Wakimoto P, Block G. Dietary intake, dietary patterns, and changes with age: an epidemiological perspective. $\mathrm{J}$ Gerontol A Biol Sci Med Sci. 2001;56(Spec No 2):65-80. 
11. Petersen KS, Clifton PM, Blanch N, Keogh JB. Effect of improving dietary quality on carotid intima media thickness in subjects with type 1 and type 2 diabetes: a 12-mo randomized controlled trial. Am J Clin Nutr. 2015;102(4):771-779.

12. Digenio AG, Mancuso JP, Gerber RA, Dvorak RV. Comparison of methods for delivering a lifestyle modification program for obese patients: a randomized trial. Ann Intern Med. 2009;150(4):255-262.
13. Paes-Barreto JG, Silva MI, Qureshi AR, Bregman R, Cervante VF, Carrero JJ, Avesani CM. Can renal nutrition education improve adherence to a low-protein diet in patients with stages 3 to 5 chronic kidney disease? J Ren Nutr. 2013;23(3):164-171.

14. Henry BW, Ozier AD, Johnson A. Empathetic Responses and Attitudes about Older Adults: How Experience with the Aging Game Measures Up. Educational Gerontology. 2011;37(10):924-941. 